\title{
RAYOSOSAURUS (SAUROPODA, DIPLODOCOIDEA) NO MESO-CRETÁCEO DO NORTE-NORDESTE BRASILEIRO
}

\author{
MANUELALFREDO MEDEIROS \\ Depto. de Biologia, UFMA, Campus do Bacanga, 65080-040, São Luís, MA, Brasil.alf@elo.com.br \\ CESAR LEANDRO SCHULTZ \\ Depto. de Paleontologia e Estratigrafia, UFRGS, 91501-970, Cx. P. 15001, Porto Alegre, RS, Brasil. \\ cesar.schultz@ufrgs.br
}

\begin{abstract}
RESUMO - Os depósitos continentais cenomanianos da Formação Alcântara no Estado do Maranhão, Nordeste do Brasil, incluem uma paleocomunidade reunindo plantas, dinossauros, crocodilos e peixes. Dentes isolados e fragmentos ósseos, principalmente centros vertebrais caudais, são os elementos mais comumente identificáveis. A comparação com um esqueleto articulado, quase completo de Neuquén na Patagônia argentina, e com dados publicados revelou que grande parte dos centros vertebrais caudais provenientes da Formação Alcântara estão relacionados a Rayososaurus tessonei (= Rebbachisaurus tessonei). Alguns destes centros também mostram similaridades com centros de "Rebbachisaurus tamesnensis" da África do norte. Apesar do confuso status taxonômico destas formas impedir uma identificação específica para a forma brasileira, esta ocorrência estabelece mais um elo entre as faunas dinossaurianas da Patagônia, do Nordeste brasileiro e do Saara.
\end{abstract}

Palavras-chave: dinossauros, Rayososaurus, Formação Alcântara, Cenomaniano, Cretáceo.

ABSTRACT - RAYOSOSAURUS (SAUROPODA, DIPLODOCOIDEA) IN THE MID-CRETACEOUS OF NORTHEASTERN BRAZIL. Continental Cenomanian deposits of the Alcântara Formation at Maranhão state, Northeastern Brazil, include a paleocommunity assembling plants, dinosaurs, crocodiles, and fishes. Isolated teeth and bone debris, mainly caudal vertebral centra, are the most common identifiable elements. Comparison with an articulated, almost complete, skeleton from Neuquén in Argentinean Patagonia, and with published data revealed that most of the caudal centra from Alcântara Formation are related to Rayososaurus tessonei (= Rebbachisaurus tessonei). Some of them also show similarity with "Rebbachisaurus tamesnensis" centra from North Africa. Although the confuse taxonomic status of these taxa precludes an accurate identification for the Brazilian form, this occurrence adds a link among the middle Cretaceous dinosaurian fauna from Patagonia, Northeastern Brazil and Sahara.

Key words: dinosaurs, Rayososaurus, Alcântara Formation, Cenomanian, Cretaceous.

\section{INTRODUÇÃO}

Os depósitos Itapecuru ocorrem em uma ampla área na região norte do Estado do Maranhão e são aflorantes, principalmente, nos barrancos de rio da bacia do Grajaú, no interior do Estado, e nas falésias litorâneas da Bacia de São Luís, na faixa litorânea (Figura 1). O espesso pacote de rochas clásticas, antes considerado como Formação Itapecuru, foi recentemente desmembrado em várias unidades litoestratigráficas (Rossetti \& Truckenbrodt, 1997), todas representando diferentes intervalos de tempo dentro do Cretáceo.

A Formação Alcântara é dominante nos penhascos litorâneos de São Luís e Alcântara (bacia de São Luís) e seus estratos areníticos e conglomeráticos guardam um rico registro fóssil do meso-Cretáceo, particularmente da fauna dinossauriana e elementos associados. O registro de dinossauros é representado, principalmente, por centros vertebrais e dentes isolados (Medeiros \& Schultz, 2001, 2002). A datação dos sedimentos Itapecuru, realizada por análise palinológica, situa o registro em um intervalo que vai do Albiano ao Cenomaniano (Pedrão et al., 1993a,b,c). Elementos da fauna dinossauriana e peixes dulcícolas associados formam uma paleocomunidade similar à que habitou o norte africano na mesma época (Medeiros, 2001; Medeiros \& Vilas Bôas, 1999; Medeiros \& Schultz, 2001, 2002).

$\mathrm{O}$ presente trabalho descreve materiais esqueletais fragmentários e desarticulados coletados no afloramento Laje do Coringa (Formação Alcântara, Ilha do Cajual, norte do Maranhão) comparáveis a Rayososaurus tessonei (Calvo \& Salgado, 1995) e a "Rebbachisaurus tamesnensis" Lapparent (1960), ambos do meso-Cretáceo, e discute as implicações da 


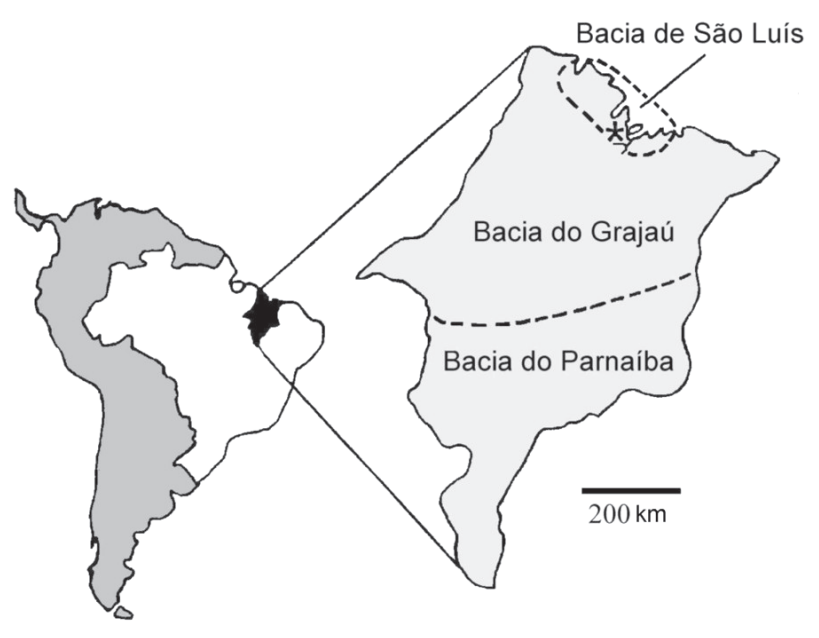

Figura 1. Bacias de São Luís e Grajaú no Estado do Maranhão, onde afloram rochas cretáceas, incluindo os depósitos Itapecuru, indicando a localização da Ilha do Cajual (asterisco) (baseado em Góes \& Rossetti, 2001).

Figure 1. São Luís and Grajaú basins in Maranhão State, where Cretaceous rocks, including the Itapecuru deposits, crops out, with the location of Cajual island (asterisk) (based on Góes \& Rossetti, 2001).

ocorrência deste material fóssil no norte-nordeste brasileiro.

Os elementos fósseis descritos estão depositados na coleção paleontológica da Universidade Federal do Maranhão (UFMA), em São Luís.

\section{SISTEMÁTICAPALEONTOLÓGICA}

\author{
DINOSAURIA Owen, 1842 \\ SAUROPODA Marsh, 1878 \\ DIPLODOCOIDEA Marsh, 1884
}

Rayososaurus Bonaparte, 1996

(Figuras 2, 3)

Material. UFMA 1.10.168; UFMA 1.10.188; UFMA 1.10.806; UFMA 1.10.015; UFMA 1.10.283; UFMA 1.10.170; UFMA 1.10.306; UFMA 1.10.728; UFMA 1.10.983; UFMA 1.10.1040; UFMA 1.10.1530; UFMA 1.10.169; UFMA 1.10.1802; UFMA 1.10.951; UFMA 1.10.990; UFMA 1.10.1252; UFMA 1.10.198.

Descrição. O centro 1, UFMA 1.10.168 (124 mm de comprimento, $85 \mathrm{~mm}$ de largura, $53 \mathrm{~mm}$ de altura); o centro 2, UFMA 1.10 .188 ( $133 \mathrm{~mm}$ de comprimento, $62 \mathrm{~mm}$ de altura, aproximadamente 76mm de largura); 3, UFMA 1.10.806 (incompleto, $116 \mathrm{~mm}$ de comprimento, 103,5 mm de largura na base, $91 \mathrm{~mm}$ de altura); e o centro 4, UFMA 1.10.015 (148,5 mm de comprimento, $86 \mathrm{~mm}$ de altura, $122 \mathrm{~mm}$ de largura) mostram padrões morfológicos similares. Todos apresentam uma constrição na região central; faces articulares que vão de bastante cavadas (centro 1) a levemente côncavas (centros 2, 3, 4); e margem ventral, em vista articular, retilínea (1) ou levemente côncava (centros 2, 3, 4). Os centros 1,2 e 3 possuem a sutura do arco neural desviada para a porção mais anterior do corpo vertebral; o centro 4 mostra uma posição mediana do arco neural. O centro 1 apresenta, em vista anterior e posterior, as margens laterais e dorsal delineando uma borda contínua e arredondada. A face ventral é plana, mas outros centros de mesma morfologia analisados apresentam uma depressão central muito rasa, antero-posteriormente orientada, similar àquela observada nos centros 2,3 , e 4 . As faces articulares dos centros 2 e 3 são sub-triangulares; as do centro 4 têm as margens laterais e dorsal mais arredondadas.

Diferente dos anteriores, o centro 5 (UFMA 1.10.283, 91 $\mathrm{mm}$ de comprimento, $126 \mathrm{~mm}$ de largura, $122 \mathrm{~mm}$ de altura) mostra uma forma geral arredondada, em vista anterior. As faces laterais possuem, cada uma, um pleurocelo profundo imediatamente abaixo da linha de sutura do arco neural. As faces articulares possuem uma leve concavidade. Um outro centro analisado, UFMA 1.10.198, tem morfologia similar, mas está mais deteriorado.

Do total de 17 elementos vertebrais analisados sete são do tipo morfológico 1, três do tipo 2, quatro do tipo 3, um do tipo 4, e dois do tipo 5 .

Discussão. A comparação do material aqui descrito com o esqueleto articulado de Rayososaurus tessonei (Calvo \& Salgado, 1995) do Museo de la Universidad del Comahue, em Neuquén, Patagônia Argentina, revelou uma considerável similaridade, permitindo definir, aproximadamente, a posição relativa dos elementos vertebrais em sequência.

Analisando os elementos vertebrais considerados caudais medianos e distais (Figura 3) é possível observar, em vista articular, uma variação progressiva da condição mais arredondada das margens laterais e dorsal (centro 4) para sub-triangular (centros 2, 3) voltando a mais arredondada (centro 1), mas sempre mantendo a margem ventral levemente côncava ou retilínea. Há também uma progressiva diminuição da altura dos corpos vertebrais, até tornarem-se bastante achatados dorso-ventralmente nos elementos mais distais.

A face ventral com uma depressão rasa, anteroposteriormente orientada, é comum a UFMA 1.10.015, UFMA 1.10.806, UFMA 1.10.188, e outros centros da amostra similares a UFMA 1.10.168, mostrando que esta característica mantem-se ao longo da região mediana e distal da cauda (Figura 3), sendo também observada nas vértebras caudais de Rayososaurus tessonei.

O centro UFMA 1.10.806 é considerado de posição mediana-distal na cauda; UFMA 1.10.188 ocuparia uma posição um pouco mais distal; e UFMA 1.10.168 localizaria-se próximo à extremidade distal da cauda do animal (Figura 3). UFMA 1.10.015 é aqui tentativamente considerado como um elemento de posição mediana a mediana-distal na cauda, anterior a UFMA 1.10.806, principalmente com base no comprimento de seu canal neural, que ocupava quase toda a face dorsal.

Um outro elemento desarticulado e fragmentário muito freqüente entre os fósseis da Laje do Coringa é um espinho neural apresentando um padrão cujas lâminas formam um desenho em cruz, em vista dorsal, algo semelhante aos espinhos neurais de vértebras dorsais e primeiras caudais de $R$. tessonei. 
Dentre o material descrito, os centros UFMA $1.10 .283 \mathrm{e}$ UFMA 1.10.198 podem não estar relacionados a $R$. tessonei. Estes centros apresentam forma e dimensões comparáveis àquelas dos elementos caudais proximais da espécie argentina, porém, apresentam pleurocelos que estão ausentes naquela espécie. Além disso, os centros vertebrais coletados na Formação Alcântara não mostram o sulco ventral profundo presente nas vértebras caudais anteriores de $R$. tessonei. Estes elementos são aqui apenas tentativamente considerados como caudais anteriores e referidos à forma brasileira cujas caudais medianas e distais estão acima descritas. Curiosamente, estes dois centros são também comparáveis às vértebras descritas e atribuídas ao "saurópode A" por Lapparent (1960: 34). No referido trabalho, o autor descreve (sem ilustrar) duas vértebras caudais anteriores em forma de disco achatado, uma delas medindo 13 X 13,5 $\mathrm{cm}$ de diâmetro, com um comprimento de apenas $6 \mathrm{~cm}$, mas não faz referência a pleurocelos. Com base nesse material e em outras peças foi estabelecida uma nova espécie, Rebbachisaurus tamesnensis Lapparent, 1960. Esta designação taxonômica foi posteriormente questionada (Calvo \& Salgado, 1995). UFMA 1.10.188 também é curiosamente comparável ao elemento caudal distal do "saurópode A", figurado por Lapparent (1960, plate VII, fig. 8). Estas semelhanças indicam que o material aqui descrito pertenceria a uma forma relacionada a Rayososaurus tessonei e ao "saurópode A" africano.
Exceto pela espécie tipo, Rebbachisaurus garasbae Lavocat, 1954, a definição do status taxonômico das espécies de Rebbachisaurus é problemática. Rebbachisaurus tamesnensis foi desconsiderado como pertencente ao gênero Rebbachisaurus por Calvo \& Salgado (1995); enquanto Rebbachisaurus tessonei foi tranferido para o gênero Rayososaurus por Wilson \& Sereno (1998).

No que diz respeito aos registros brasileiro e norte-africano, uma definição taxonômica precisa depende da eventualidade de encontrar-se material em melhor estado de conservação dos dois lados do Atlântico Sul. Tanto os elementos vertebrais da Formação Alcântara (Nordeste brasileiro) quanto os do Continental Intercalar do Saara ("Rebbachisaurus tamesnensis") são dissociados e, em sua maioria, deteriorados. É importante frisar que as vértebras caudais de Rebbachisaurus garasbae não são conhecidas.

Diante deste quadro confuso, pode-se afirmar, por ora, tão somente que o táxon encontrado no norte maranhense mostra similaridade com as formas atribuídas a Diplodoicodea tanto no norte africano quanto na Patagônia Argentina. Considerando o material coletado até agora, esta similaridade é maior em relação ao táxon argentino, Rayososaurus tessonei. A grande freqüência destes elementos vertebrais, particularmente dos centros tipo 1, aqui considerados caudais distais (UFMA 1.10 .168 e similares), sugere que o táxon aqui registrado ocorria em número expressivo na comunidade dinossauriana do Eocenomaniano do norte-nordeste brasileiro.
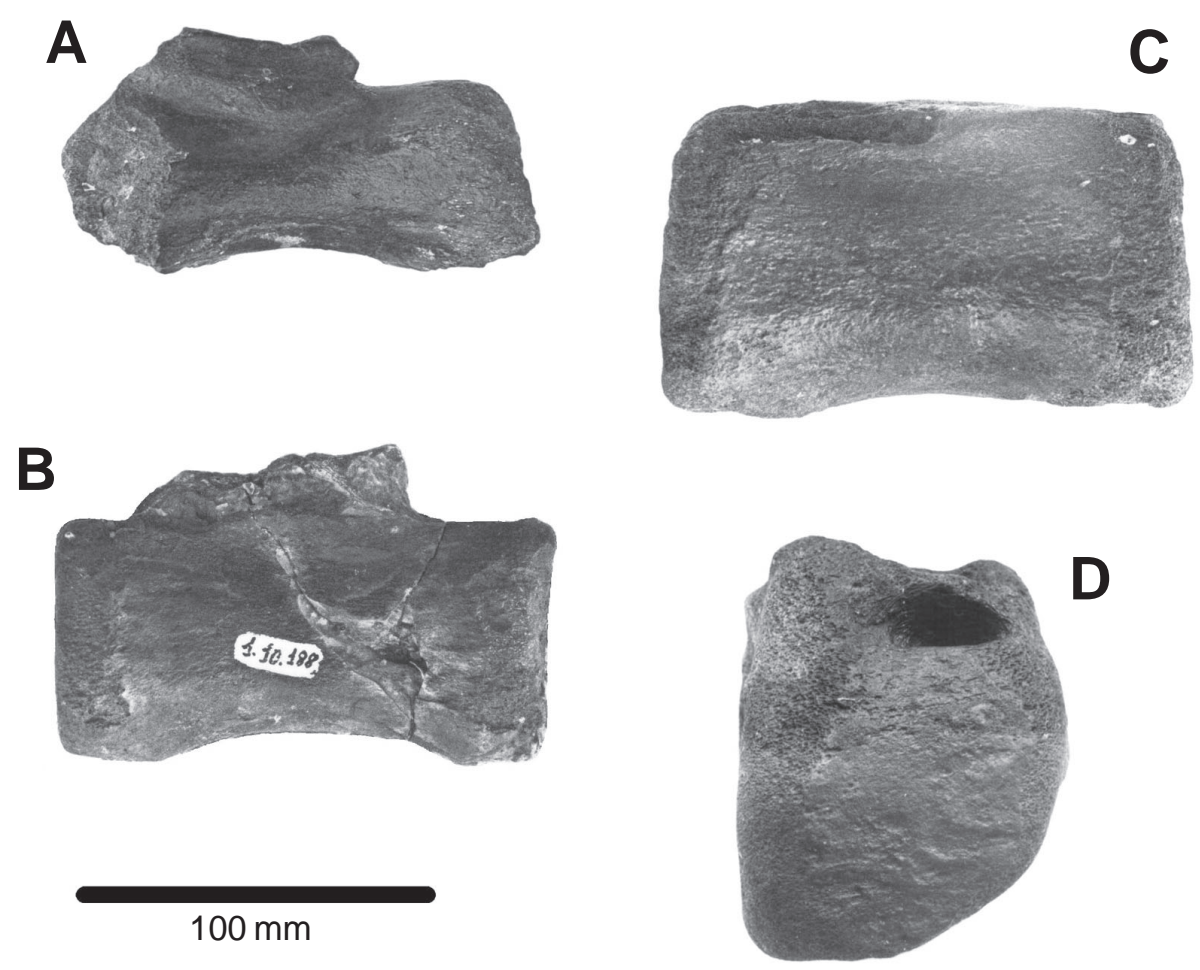

Figura 2. Centros vertebrais caudais coletados na Laje do Coringa: A. UFMA 1.10.168; B. UFMA 1.10.188; C. UFMA 1.10.015; D. UFMA 1.10.283; A-C em vista lateral esquerda; D. provavelmente em vista lateral esquerda.

Figure 2. Vertebral caudal centra collected at Laje do Coringa site: A. UFMA 1.10.168; B. UFMA 1.10.188; C. UFMA 1.10.015; D. UFMA 1.10.283; A-C in left lateral view; D. probably in left lateral view. 


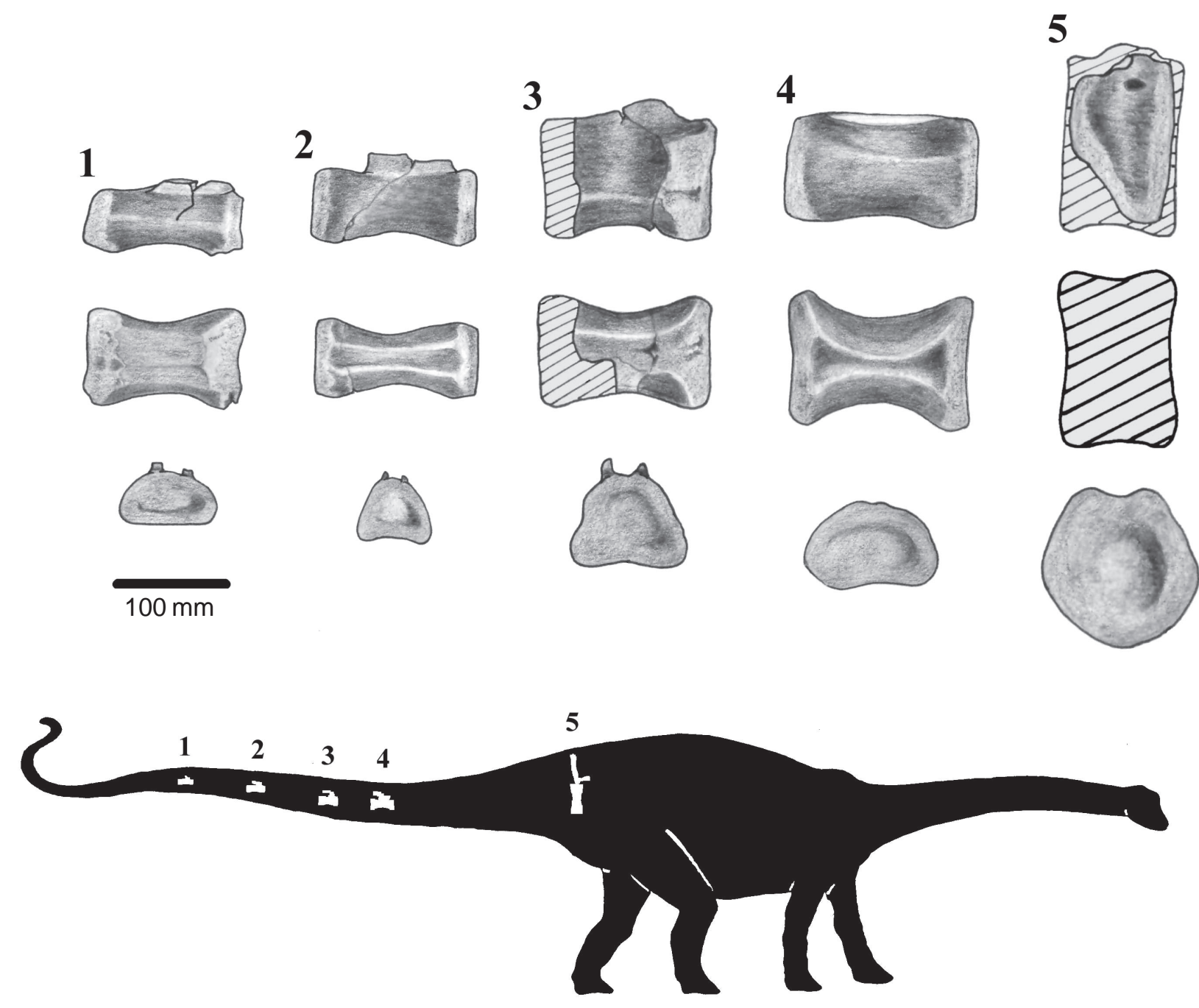

Figura 3. Centros vertebrais caudais atribuídos a Rayososaurus em vista lateral direita (linha superior), ventral (linha mediana), e articular (linha inferior) e sua provável posição relativa na cauda. 1. UFMA 1.10.168, 2. UFMA 1.10.188, 3. UFMA 1.10.806, 4. UFMA 1.10.015, 5. UFMA 1.10.283. Na fila inferior: $1 \mathrm{em}$ vista posterior, 2 e 3 em vista anterior, 4 e 5 provavelmente em vista anterior.

Figure 3. Caudal vertebral centra assigned to Rayososaurus in right lateral (top line), ventral (middle line), and articular (bottom line) views and their probable relative position in the tail. 1. UFMA 1.10.168, 2. UFMA 1.10.188, 3. UFMA 1.10.806, 4. UFMA 1.10.015, 5. UFMA 1.10.283. In the lower line: 1 in posterior view, 2 and 3 in anterior view, 4 and 5 probably in anterior view.

Centros caudais isolados, desarticulados de seus respectivos complexos neurais são pouco utilizados como elementos diagnósticos. Porém, quando se dispõe somente destes elementos para a identificação, um esforço tem que ser feito para realizar um diagnóstico efetivo. Pelas características da gênese da Laje do Coringa, interpretada como tendo se dado em um contexto marinho raso, que reuniu elementos retrabalhados (Holz et al., 2001) de diferentes fontes, com pelo menos algumas delas de origem fluvial (Medeiros, 2001), não foi possível, até agora, coletar material de dinossauros mais diagnóstico que os dentes e centros vertebrais isolados. Estes elementos têm, em muitos casos, uma utilidade limitada nas diagnoses. Entretando, com a grande quantidade e variedade de centros caudais encontrados no referido afloramento é possível distinguir características que os relacionam entre si, bem como com outras ocorrências contemporâneas em outras regiões. O mais difícil é definir o real grau de afinidade entre os táxons aparentados de diferentes regiões. Neste caso, informações indiretas são úteis. Um critério que tem sido usado, em caráter tentativo, como aproximador do parentesco entre os táxons da Formação Alcântara e aqueles do norte da África é a semelhança do conjunto faunístico, tanto de dinossauros como de outros vertebrados associados, em um intervalo dentro do Albiano-Cenomaniano. A mesma associação de dentes de Carcharodontosaurus Stromer, 1931e Spinosaurus Stromer, 1915 comum nos depósitos do Saara ocorre na Formação Alcântara. A fauna africana, composta pela tartaruga Araripemys Price, 1973, peixes como o celacantídeo Mawsonia Mawson \& Woodward, 1907, e os dipnóicos Neoceratodus africanus Haug, 1905 e Asiatoceratodus cf. A. tiguidiensis (Tabaste, 1963), além de outros elementos esqueletais de posição taxonômica discutível como Sigilmassasaurus Russell, 
1996, e o material atribuído a Astrodon sp. descrito e figurado por Lapparent (1960) é bastante similar àquela existente no Brasil (Medeiros, 2001; Medeiros \& Schultz, 2001, 2002; Dutra \& Malabarba, 2001; Cunha \& Ferreira, 1980). Se a composição faunística como um todo é muito semelhante, então as paleocomunidades do norte-nordeste brasileiro e norte africano deveriam manter um grau de similaridade, com diferenciação apenas a nível específico.

A fauna albiana-cenomaniana do norte-nordeste brasileiro parece mais assemelhada à norte africana que à sulamericana austral (Medeiros \& Schultz, 2002). Os elementos descritos no presente trabalho levantam novas sugestões sobre a fauna da Fomação Alcântara e suas relações com a africana e a patagônica, apesar de sua natureza fragmentária. Em termos gerais, este registro estabelece mais um elo, embora ainda vago, entre a fauna dinossauriana do norte-nordeste brasileiro, aquela do extremo sul do continente americano e a do norte africano.

\section{AGRADECIMENTOS}

Agradecemos aos alunos do Curso de Ciências Biológicas da UFMA pela decisiva ajuda na coleta do material fóssil da Laje do Coringa. Agradecemos especialmente a Leonardo Salgado pelo acesso ao material fóssil de Rayososaurus tessonei no Museo de la Universidad del Comahue, em Neuquén, Argentina. Este trabalho teve o suporte financeiro da UFMA, CAPES e UFRGS.

\section{REFERÊNCIAS}

Calvo, J.O. \& Salgado, L. 1995. Rebbachisaurus tessonei sp. nov. a new sauropoda from the Albian-Cenomanian of Argentina; new evidence on the origin of the Diplodocidae. Gaia, 11: 13-33.

Cunha, F.L.S. \& Ferreira, C.S. 1980. Um dipnoi na Formação Itapecuru (Cenomaniano), Maranhão, Brasil. In: CONGRESO ARGENTINO DE PALEONTOLOGIA Y BIOESTRATIGRAFIA, 2, 1980. Actas, Buenos Aires, 2: 1-9.

Dutra, M.F.A. \& Malabarba, M.C. S. L. 2001. Peixes do AlbianoCenomaniano do Grupo Itapecuru no estado do Maranhão, Brasil. In: D. F. Rossetti; A. M. Góes \& W. Truckenbrodt (eds.) O Cretáceo da Bacia de São Luís-Grajaú, Museu Emílio Goeldi, p. 191-208.

Góes, A.M. \& Rossetti, D. 2001. Gênese da Bacia de São LuísGrajaú, Meio-Norte do Brasil. In: D.F. Rossetti; A.M. Góes \& W. Truckenbrodt (eds.) O Cretáceo na Bacia de São Luís Grajaú, Museu Emílio Goeldi, p. 15-29.
Holz, M., Medeiros, M.A. \& Schultz, C.L. 2001. Tafocenoses de vertebrados formando resíduos transgressivos - o exemplo do sítio fossilífero Laje do Coringa do Neocretáceo da Bacia de São Luís, no norte do Brasil. In: CONGRESSO BRASILEIRO DE PALEONTOLOGIA, 17, 2001. Boletim de Resumos, Rio Branco, UFAC, p. 36.

Lapparent, A.F. de 1960. Les dinosauriens du continental intercalaire du Sahara central. Memoires de la Société Géologique de France, N.S. 88A: $3-56$.

Medeiros, M.A. 2001. A Laje do Coringa (ilha do Cajual, bacia de São Luís, baía de São Marcos, MA): conteúdo fossilífero, bioestratinomia, diagênese e implicações na paleobiogeografia do Mesocretáceo do nordeste brasileiro. Programa de Pós-Graduação em Geociências, Universidade Federal do Rio Grande do Sul, Tese de Doutorado, $134 \mathrm{p}$.

Medeiros, M.A. \& Vilas Bôas, I. 1999. Ocorrência de uma paleocomunidade continental do Cenomaniano (Cretáceo Superior) do Nordeste do Brasil. In: JORNADAS ARGENTINAS DE PALEONTOLOGIA DE VERTEBRADOS, 15, 1999. Resumenes, La Plata, UNLP, p. 18.

Medeiros, M. A. \& Schultz, C. L. 2001. Uma paleocomunidade de vertebrados do Cretáceo Médio, bacia de São Luís. In: D.F. Rossetti; A.M. Góes \& W. Truckenbrodt (eds.) O Cretáceo na Bacia de São Luís - Grajaú, Museu Emílio Goeldi, p. 209221.

Medeiros, M.A. \& Schultz, C.L. 2002. A fauna dinossauriana da Laje do Coringa, Cretáceo médio do Nordeste do Brasil. Arquivos do Museu Nacional, 60(3): 155-162.

Pedrão, E.; Arai, M.; Barrilari, I.M.R. \& Carvalho, I.S. 1993a. Análise palinológica de uma amostra de superfície de Querru (Formação Itapecuru), Município de Itapecuru-Mirim - MA. Rio de Janeiro, PETROBRAS, CENPES, 11 p. (Relatório Técnico).

Pedrão, E.; Arai, M.; Carvalho, I.S. \& Ferreira, C.S. 1993b. Palinomorfos de sedimentos albianos (Formação Itapecuru) da Bacia do Parnaíba. Rio de Janeiro, PETROBRAS, CENPES, 13 p. (Relatório Técnico).

Pedrão, E.; Arai, M.; Carvalho, I.S. \& Santos, M.H.B. 1993c. Palinomorfos da Formação Itapecuru - análise palinológica de uma amostra de superfície da Ponta do Farol, São Luís MA. Rio de Janeiro, PETROBRAS, CENPES, 10 p. (Relatório Técnico).

Rossetti, D. de F. \& Truckenbrodt, W. 1997. Revisão estratigráfica para os depósitos do Albiano - Terciário Inferior (?) na bacia de São Luís (MA), norte do Brasil. Boletim do Museu Paraense Emílio Goeldi (Série Ciências da Terra), 9:29-41.

Wilson, J.A. \& Sereno, P. 1998. Early evolution and higher-level phylogeny of sauropod dinosaurs. Journal of Vertebrate Paleontology, 18(2, suppl.):1-68. 
